Article

\title{
Analyses and Optimization Methods of Legged Robots: A review
}

\author{
Venkata Nandam ${ }^{1}$, Praveen Seelaboyina ${ }^{2}$, Sandeep Chowdary ${ }^{3}$, Hari Krishna Molleti ${ }^{4}$ \\ ${ }^{1}$ Department of Mechanical Engineering,Lovely Professional University,Punjab,144411,India. \\ 2Department of Mechanical Engineering,Lovely Professional University,Punjab,144411,India. \\ ${ }^{3}$ Department of Mechanical Engineering,Lovely Professional University,Punjab,144411,India. \\ ${ }^{4}$ Department of Mechanical Engineering,Lovely Professional University,Punjab,144411,India.
}

\begin{abstract}
During the most recent years, a lot of research has been done in creating robots with more selfgovernance so that they can overcome the challenges that real world environments present. The robot's limited versatility in real world applications can be overcome by the development of Legged robots. Also, as they permit movement in unavailable territory to robots with wheels, Legged Robots are more advantageous. But the potency of the legged robots explicitly its energy usage among alternate points of view really fall behind robots that use wheels. So, the present status of development, there are as yet a few perspectives that need to be analysed, optimized and enhanced. This paper presents review of literature of various biologically inspired legged robots, various techniques adopted for their analysis and optimization and the analysis and optimization of the ones that are not biologically inspired
\end{abstract}

Keywords: Legged robots, Optimization, Kinematic analysis, Compliant Joints.

\section{Introduction}

Nowadays, in general public where development affects everything and each zone of human information is upheld and enhanced by utilizing innovation,robotics has a focal part in our lives. Robots that can move, have numerous operations from military to space investigation, from mechanical use,to execution in day by day life. Relying upon their design,there are two principle parts of mobile robots:Legged and wheeled. Legged robots present critical preferences over customary robots having wheels and tracks. Wheeled robots request cleared surfaces for mobilization, being very quick and compelling in them.Similarly, these components can be uncomplicated and be of less weight.Anyway over half of the earth surface is blocked off to conventional robots being troublesome or even impossible that they manage enormous snags and surface unevenness. Even all the landscape models can just outperform little obstructions and surface irregularities, but it involves utilization of high amount of energy.As to legged robots,although they present expanded versatility in troublesome terrains, they can't outperform a considerable lot of the challenges and their energy utilization is moderately high.In addition to these problems,one must also consider the way the customary robots leave non stop trenches on the ground, which in certain circumstances is disadvantageous. We can use these legged robots for salvage work after seismic tremors and in perilous places, for example, within an atomic reactor,giving them incredible potential.

Based on what we saw, it is conceivable to presume that legged frameworks offer a predominant portability in normal terrains,as these robots utilize several grips for each foot,contrary to wheeled robots which need constant help surface. Hence, these robots can move in unpredictable territories by shifting their legs design to adjust to anomalies on the surface.Besides this, the feet may set up contact with ground in chosen points as per the territory conditions.For these reasons legged ones are intrinsically sufficient frameworks for motion in unpredictable terrain. When these robots move on delicate land coverings, as for example on sandy soil,the capacity for utilization of several adhesive forces on the ground can likewise enhance how much energy is consumed,as they disfigure the territory less than wheeled or tracked robots. Hence,the energy expected for 
escaping depressions is lesser and we can make a contact zone between foot and ground,so that the ground uphold pressure is little.In addition the utilization of various DOF in legged joints permits these robots to change where they head without any slippages.It is likewise conceivable to differ the height of the body,introducing a dumpling and a decoupling impact between landscape anomalies and the robot frame.Now concerning locomotion, it should likewise be referenced the likelihood that these frameworks can embrace themselves to surfaces on which they exhibit motion. This is especially evident for cases where they move over the external surface of pipes, so as to build their equilibrium capacities.

Albeit legged robots offer all these possible benefits, in the present status of research and development,there are a few viewpoints which must be enhanced.Considering this thought a few optimization procedures have been suggested and enforced to these frameworks, either during their design and construction or during their operation.Among the enhancement standards followed by particular creators,one may incorporate viewpoints identified with energy,efficiency,stability, velocity,comfort,mobility and ecological effect.

This paper reviews various methods and approaches that have been used for optimizing the structure and locomotion modes of unnatural legged systems. The rest of this paper is structured as follows, Section two discusses some analysis and optimization done in various recent biologically inspired robots and is subdivided into biped,quadruped and hexapod robots,Section three discusses the same but of the ones that are not biologically inspired.At the end,Section four presents the important outcomes of this study.

\section{Bio Inspired Robots:}

During a huge number of years,evolution has made entrancing methods of traveling through a wide range of natural habitats. The most interesting types of earthbound headway are walking,running and jumping. Numerous animals and insects have achieved perfection in their type of locomotion like the extraordinary gecko that climbs. Researchers and architects have been noticing creature headway for many years and are as yet entranced and are attempting to uncover nature's mysteries everywhere on the earth. Thus, designers of legged mechanical frameworks attempt to mimic nature's amazing development and coordination standards. These days one can discover naturally roused mechanical frameworks in practically all fields of automated examination.

As legged robots are propelled among creatures seen in nature,a successive methodology for designing them and constructing them to build a robotic copy of the creature which is proposed to be imitated,either in terms of physical dimensions or in terms of characteristics such as gait and actuation of limbs is proposed.Considering this target,definite investigations of the anatomy and locomotion of the life systems of various creatures have been proposed.Researches that join specialists from biology and robotics are frequently introduced.

\subsection{Biped/Humanoid Robots:}

From antiquated times, man has attempted to make mechanisms that look like the human body. One of the goals of present robotic research is to create machines with more self governance for managing difficulties of certifiable conditions. The machine's restricted flexibility is by all accounts a significant hindrance to utilize them in genuine applications.Particularly in jumbled surroundings, excess equipment configuration is a conceivable outcome for broadening the robot's versatility.Humanoids are one unmistakable agent of excess versatile robots.Owing to their repetitive kinematic structure,these robots are foreordained for utilization in different complicated situations.Generation of motion for humanoids usually follows an ordered approach: First,task space directions are produced to empower powerfully achievable movements dependent on improved prototypes and models over a long period of time.Next,the machine's reverse kinematics is comprehended topically when abusing the redundancy for secondary optimization targets.So the overall motion depends on the factors that influence the characteristics of the structures and functions of the robot and also those connected to physical phenomena such as friction,gravity and reaction forces. Humanoid biped machines are useful in all stages of a man's daily life. These robots will be made to work in clinics,services and in homes and we can expect them to be involved in sports.

Among the enormous number of models receiving this methodology,one can make reference to the following robots: 
- The BIP robot that presents the kinematic and dynamic parameters similar to the anthropomorphic values of a human,with $90 \mathrm{~kg}$ mass and a height of 1.7m (Espiau,1997);

- The biped robot presented by Caldwell,et al.(1997) for testing actuation using artificial pneumatic muscles rather than actuators.

- The WABIAN humanoid,whose joint range of motion and size are based on adult human (Yamaguchi and Takanishi 1998);

- The Honda Humanoid Robot model P2 displayed the joint locations, dimensions,center of gravity and ranges of motion similar to those of human leg(Hirai,1998);

- The BIP2000 robot with kinematic and dynamic parameters similar to those of a man, with a height of 1.8 m.(Espiau and Sardain,2000);

- The Robian II,with weights and dimensions close to those of an average European man(Gravez,2002);

- The Johnnie which displays a geometry similar to that of a male human body with a height of $1.8 \mathrm{~m}$ (Lohmeier,2004).

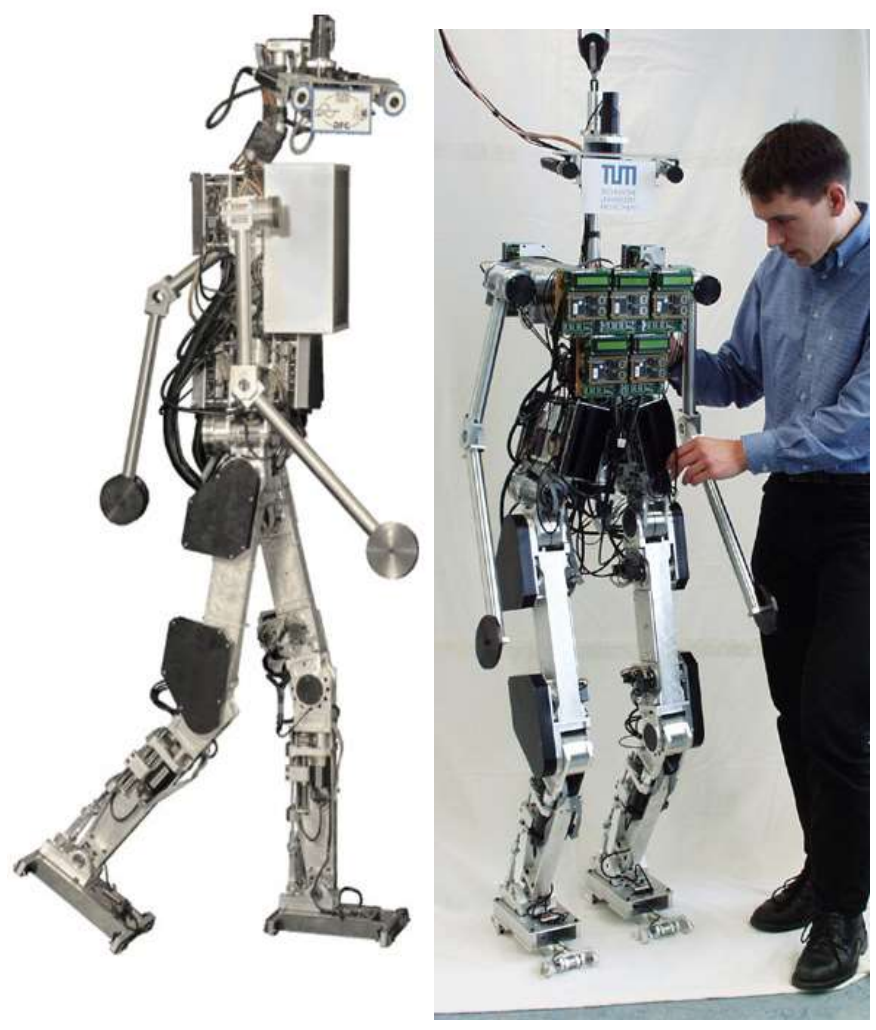

Biped Johnnie Robot

Now the discussion will be focused on what progress in the research associated with those of biped robots has been done in the recent past.

Marco Ceccarelli[1],et.al.,(2012) introduced an activity investigation of models having Chebyshev and Pantograph linkage structures which are intended to be applied in minimal effort simple activity humanoid robot.The issues that emerge from ordinary biped robots, for example, complex design, more number of engines and their legitimate distribution,sophisticated control calculations and power clashes among engines and leg swings are dispensed by the diminished number of levels of freedom. Unlike the vast majority of the other humanoid robots, the balance of the legs brought about diminished time for walking.During simulation, it has been seen that the proportion of connection sizes of Chebyshev will extraordinarily influence the state of the directions. 
Mingfeng Wang[2],et.al., (2015) introduced a machine-driven plan of a novel Cassino biped locomotor comprising of two mount leg systems with the help of a powerful strolling simulation.To ensure feet having unadulterated translational movement , the two $U$ joints in every appendage are organized with two external revolute joints corresponding to one another and two inward revolute joint additionally corresponding to each other.Planning of step age is performed with abdomen swinging and this midriff swinging can likewise be utilized for COG position change purpose.Simulation results from MSC.ADAMS show that locomotor with appropriate info powers of direct actuators gives restricted response powers between ground and feet and has a viable and achievable strolling capacity on a leveled terrain. It has likewise been seen that the effect powers of the beginning and the ending stages are a lot bigger than those of typical two legged strolling phases, because of the change from statically stable strolling to progressively stable strolling at this instant.It has additionally been seen that equal controllers have better exhibitions regarding accuracy,dynamic performance and payload ratio to possess weight.

E.Rodriguez Leal[3],et.al., (2012) built up a mechanical framework with 7DOF for every leg and 1 DOF for each toe joint.They additionally introduced close conditions for the direct and reverse kinematics by partitioning the strolling stride into Frontal and Sagittal planes.Lagrangian elements have been utilized for numerical displaying of the dynamic conditions to give a comprehension of precarious elements related with biped stance and to empower the plan of dynamic adjustment controls for robots.A control approach utilizing a PD control law with gravity remuneration was repeated for controlling the ideal directions and locating the necessary force required by the leg joints. The arrangement of dynamic toe joint permits the robot to stroll in a more normal manner.

Giovanni Gerardo Muscolo[4],et.al., (31st March 2020) introduced the issue of compliance in biped locomotion.A biped robot with a similar connection measurements of a standard man has been planned and mimicked with altered compliance of the appended connections and afterward recreation results have been contrasted by expanding and decreasing compliance on hip,knee and lower leg joints. The simulation results show that robot exhibitions, for example, speed and force can be improved by remembering the agreeable component for the knee joint in light of the fact that the compliant joint can retain effects and move energy. The imprudent burdens to which the actuators have been oppressed during strolling and running have been limited due to the disconnection between the line of association of the connections and the actuator because of the compliance.It has additionally been assessed how much the firmness of each joint separately taken can be enhanced to limit energy.

Rahul R Thavai[5],et,al., (2015) built up a basic 11 DOF biped robot utilizing 5 links,2 joints for every leg and one for the torso that is associated through revolute joints, for example, hip,knee and lower leg joints to impersonate human motion.Kinematic model is acquired through $\mathrm{D}-\mathrm{H}$ method and Dynamic model through Lagrangian formulations. Motion arranging and Kinematic investigation have been done in MATLAB for figuring joint and strolling parameters.And the exhibition of the robot is assessed through occasional strolling simulations in Simulink.To decide corresponding and subordinate gains just as the reference plot for the hip swing, a regulator has been set up on the robot.The robot has more levels of opportunity in order to keep away from the limitation of movement in workplace.

Jose Antonio Tenreiro Machado[6],et.al., (1999) introduced kinematic examination and displaying of biped motion frameworks with an intend to decide kinematic attributes and framework execution during strolling by portraying the movement regarding five variables:hip height,step length,maximum hip ripple,maximum foot leeway and connection lengths. Four strategies have been proposed to perceptibly gauge the presentation of the machine: perturbation analysis,energy analysis,low pass recurrence reaction investigation and locomobility measurement.Simulation results show that knee joint might be a main consideration because of critical scope of execution variation.A significant debasement happens as the progression length esteem increases.The hip appeared to be especially touchy to the states of hip height.Locomobility investigation assisted with choosing a base foot freedom to keep away from unplanned contact.

Prasanth Kumar R[7],et.al., (2013) introduced kinematic examination of four connected biped robot in both position and flight stages considering the impacts of precise momentum,Zero Moment Point (ZMP) and landing stability.During position phase,constraint conditions are presented on the ZMP to guarantee soundness and the central rakish energy to make the biped robot non holonomic and to stay away from superfluous torso 
moment.During flight phase,the vertical separation between the foot and the focal point of mass is arranged with the end goal that the speed of the robotic foot arrives at null at the hour of arriving to guarantee smooth arrival with no impact.Results of simulation have been introduced for both the help and aviation stages for different introductory robotic designs which brought about variety of the level separation and vertiginous stature that the robot had.

Elvedin Kljuno[8],et.al., (2010) built up a disentangled control plan as a reverse plant model of a 10 DOF biped strolling robot that utilizes non direct stride planning,as the standard straight control configuration is related with issues of moderately convoluted architecture,high DOF, and adjusting requirements. Another hugeness of this methodology is the diminished count prerequisites because of improved system of ostensible calculation of joint forces. Kinematic and Dynamic examinations are talked about inclusion of the outcomes for ground powers and joint torques important for execution of a recommended strolling movement and correlation of test information.

\subsection{Quadruped Robots:}

Quadrupedal or four legged robots display quadrupedal movement. These robots profit by expanded strength over bipedal ones,particularly during its motion.A quadruped robot,at moderate speeds just move a single leg at a time to ensure a steady mount.Quadrupedal robots additionally advantage from a lower focus of gravity than biped systems.

Among the enormous number of models receiving this methodology,one can make reference to the following robots:

- The TITAN series developed by the Hirose-Yoneda Laboratory since the 1980 s.

- The dynamically stable BigDog,developed in 2005 by Boston Dynamics,NASA's Jet Propulsion Laboratory, and the Harvard University Concord Field Station.

- Successor of BigDog,the LS3 (2012).

- MIT's new back flipping mini Cheetah robot (2018).
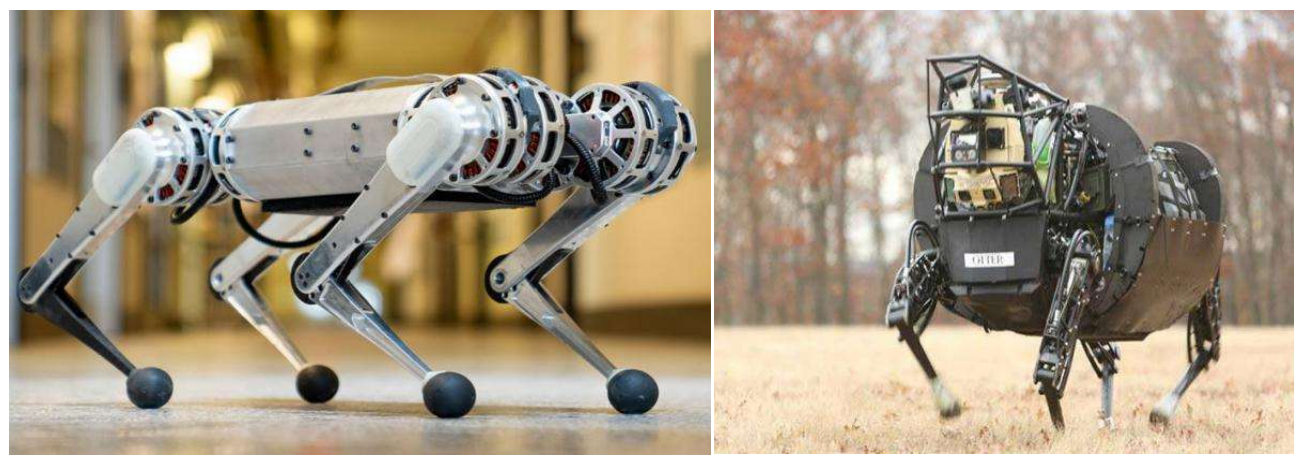

MIT's mini Cheetah

Boston Dynamics's LS3

Anand Kumar Mishra[9],et.al., (2014) did design,fabrication and stride arranging dependent on high walk corner to corner run step example of a croc propelled robot having eight levels of opportunity with every leg having two revolute joints speaking to knee and hip individually that are incited by servo motors. Headway control is finished with end effector having a setup of every one of four legs as a function of servo angles.LAEE (Limb Angles to End Effector) and SALA work approach has been utilized to decide those servo functions.To actualize the gait,paramaterization of obligation factor and stage point of every leg has been done.The obligation factor controlled the measure of point pivoted per turning. The stage boundary controlled the recurrence of venturing and turning. 
Takuma Nemoto[10],et.al., (2015) built up a reconfigurable quadruped robot motivated from huntsman creepy crawly that can perform strolling and moving movement and change between strolling and moving by reconfiguring its legs.To make up for robot's energy misfortune during locomotion, a regulator has been planned dependent on a unique model of the quadruped robot.This dynamic model depicts motion along planes and is then applied for figuring mechanical energy in mathematical recreations from which we can acquire the check of the adequacy in accomplishing intermittent moving headway of the proposed controller.This dynamic model additionally produces joint force for its supporting leg which permits machine's motor energy to arrive at a target energy.

Tan-Phuc Lee[11],et.al., (2015) built up a reconfigurable Klann instrument based four legged strolling robot whose exhibition attributes have been evaluated.This plan of robots is related with straightforwardness of step control,the capacity to produce different strides by basic system changes and decrease in regulator multifaceted nature and energy to drive actuators.A capricious methodology dependent on one level of opportunity was introduced and it requires incitation at simply one joint to acknowledge strolling including various legs through planning of inside cyclic walks into circular ones.Various walks to navigate beyond what one landscape can be created by applying parametric changes to this Klann component.

Giovanni Gerardo Muscolo[12],et.al., (07 May 2020) examined the conduct of a quadrupedal robot established by one dynamic Degree Of Freedom in every leg and one latent Degree Of Freedom in each spine and knee with an expectation to build the movement exhibitions of the robot by changing the stiffness of the springs.Simulations have been performed from which they demonstrated how the spine's firmness impacts the presentation of the machine by speeding up and lessening the energy needed by servo motors.Comparison has been done between agreeable legs and spline and unbending legs and spine.It has been seen that the pinnacle force produced by the front leg is twice that of the back one.It has additionally been seen that presenting a compliance in the robot will reallocate more amount of energy in various actuators.

Yunquan Li[13] et.al., Bioinspir Biomim (2019) introduced an examination on progress of quadrupedal robots enlivened by canids or felids, for example, presenting canine rowing system.Hydrodynamic drag power investigation was directed for a model automated canine which was created utilizing four precharged pneumatic actuators with contemplations of relative places of Center of Gravity and Center of Buoyancy and their dynamic variety in paddling.It was discovered that such delicate actuators have an incredible potential in the improvement of land and/or water capable legged robots as they are innately against rusty, water tight,simple in basic plan and have huge hydrodynamic preferred position due to their general semi-round and hollow shape.

Fan Jizhuang[14] et al. Bioinspir Biomim (2020) gave research on organically motivated self contained,untethered mechanical frog that can swim deftly and proficiently for many minutes in water by impersonating the rowing stride of the common frog. Two sorts of pneumatic actuators have been planned and joined to automated frog legs to make them lighter and more compact.It is discovered that such delicate actuators have incredible potential in creating land and/or water capable robots since they are quick responding,inherently water tight and basic in auxiliary design.And the mechanical frog is found to go with high natural versatility and upgraded swimming execution.

Maria Marietto[15],et.al., (2017) created TB-Horse II model roused from the organic highlights in the pony breed Mangalarga Marchador.The horse stride was actualized and approved utilizing the Virtual Robot Experimentation Platform (V-REP).This robot with horse life structures is appeared to have significant preferences over regular versatile robots, for example, working in various sorts of conditions.

\subsection{Hexapod robots:}

Hexapods or Six legged robots are roused by a longing for much more prominent dependability than bipedal or quadrupedal robots. Their last models often copy the logistics and working of bugs and similarly their gaits may be differentiated.

These include:

- Wave Gait: the slowest gait,in which pairs of legs move in a "wave" from the rear to the front 
- Tripod Gait: A slightly quicker step,in which three legs can move at once. The remaining three legs provide stability for the robot.

Among the enormous number of models receiving this methodology, one can make reference to the following robots:

- Odex,a 375 pound six legged robot developed by Odetics in the 1980s. Odex has onboard computers that control each leg.

- Genghis,one of the simplest and the earliest autonomous hexapods, developed at MIT by Robert Brooks in the 1980s.

- The modern toy series, HexBug (2007)

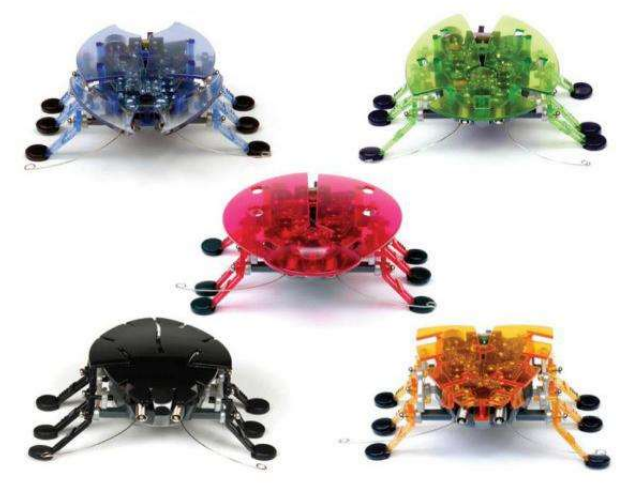

HexBug

Giuseppe Carbone[16],et.al., (2009) built up a novel organic mount strolling robot propelled by the stand strides existing in the nature.The issues related with mechanical plan are researched and the necessities of the legged component to have legitimate mount strolling step are considered.The proposed model is a diminished DOF leg instrument to ensure that the control calculation is disentangled and even not required as the system can be minimal and robust.In request to dodge power struggle between legs, an arrangement has been proposed with two legs on the ground delivering a straight line movement in level plane without waving in vertical direction and with same speed.To recommend facilitated movement of three leg mechanisms, an arrangement has been proposed where the incitation speed of the info wrench will be kept twice,during swinging stage and the supporting stage so that the two legs will consistently have a contact with the ground and perform different fluctuations in the air.Based on the reenactment results, it was discovered that an adjusting mechanism,most likely a four bar linkage, with appropriate mass at its end,can be introduced on the robot's body to change the weight community between the two legs.As an outcome, stand strolling robot can stroll with a static harmony due to basic control calculation and extraordinarily estimated adjusting system. 


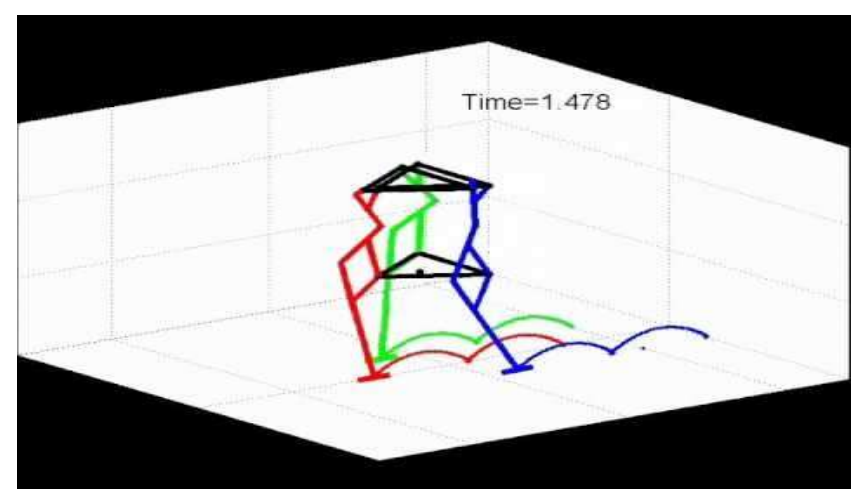

Novel Tripod Robot

Mohammadali Shahriari[17],et.al., (2014) introduced an investigation on trial six legged strolling robot model 'SiWaReL' with 18 levels of freedom. Every leg of the model has 3 DOF that is naturally roused by arachnid's leg.A servometer is utilized for each revolute joint of the robot.The legs are adjusted radially symmetric and this evenness enables the robot to walk paying little mind to the arrangement of the body.Two strolling strides mount step and wave walk have been considered and simulated.In stand gait,two symmetrical triangles have been determined,one for legs that stand and the other for legs that swing.The standing legs which are on the ground form a structure of a triangle. When the machine is pushing ahead on standing legs, the other triangle is pushing ahead over the ground to get into another position.In wave gait, the machine moves its legs individually to get to the most noteworthy solidness request but the cycle is still exceptionally moderate.

Shihbendu Shekhar Roy[18],et.al.,(2009) introduced kinematic and dynamic examinations of a hexapod robot in which a three revolute (3R) kinematic chain has been decided for every leg instrument for imitation of the leg structure of an insect.Based on position,orientation,velocity and speeding up of the storage compartment body, initial feet positions and stride pattern,joint displacements,joint velocities,joint accelerations,support feet powers and force estimations of each joint have been calculated.The direction age issue during the help stage has been detailed as a streamlining issue and comprehended utilizing the most un-squared method.LagrangeEuler definitions have been used to decide the joint forces variety over the movement cycle and for determining dynamic conditions.

Hasan Alli[19],et.al., (2007) built up a six legged robot enlivened from a cockroach created with just two actuators for driving the system. They added springs between certain connections to produce more steady strolling on unpleasant ground and copy muscles.In this layout the up/down movement of mount legs are obtained by turning both the right and left cam shafts in a similar way,utilizing only one actuator as opposed to utilizing various actuators for each leg. The single actuator plays out all the errands vital for up/down movement and rearranges the control of the system. In this examination just straight line directions were figured out.

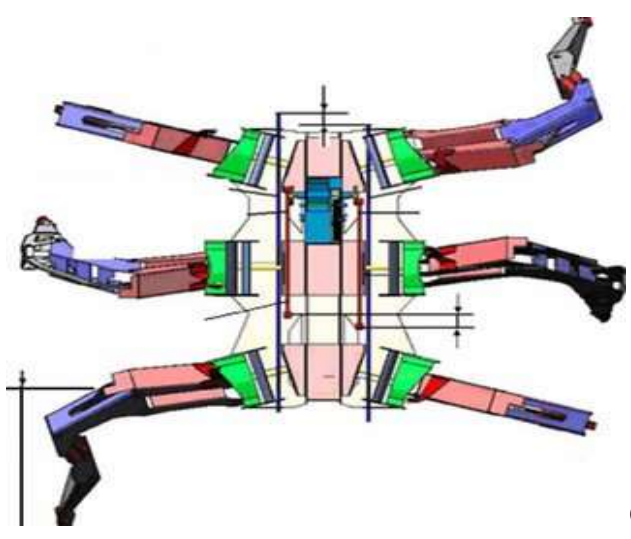

Cockroach inspired Hexapod robot 
M.Ajmal Khan[20],et.al.,(2011) introduced an investigation on kinematic examination of a mobile robot and dynamic recreation of three organically propelled strolling steps, for example, mount gait,ripple step and wave step regarding their exceptional leg consecutive movements and kinematic characteristics.A straightforward strategy for step age that empowers the machine to design its posture at various directions by using versatile leg strokes and step lengths has been described.The execution of the stride age technique has been assessed as far as rotational angles, velocities and accelerations are considered.To review following blunders, the data from the installed sensors was utilized as control criticism and a wayboard route calculation was utilized to guide the machine over a lopsided surface.Using fast prototyping,the robot has been appeared to perform speed controlled strolling to accomplish greatest leg stroke for the most extreme step length which makes the change starting with one walk then onto the next quicker.It has additionally been seen that the femur and coxa joints that are principally answerable for the precise pivot and levitation of the leg are impelled moderately slower than the tibia joint that is utilized for the augmenting the leg. Hence,the expansions of the leg are accomplished quicker to build the portability of the walk while the levitation,turning and landing of the leg are accomplished generally slower to guarantee kinematic soundness and limit the impact of imprudent powers during leg-ground associations.

G.Figliolini[21],et.al., (2007) introduced a kinematic model and total stride reenactment of the leg tip of a hexapod strolling machine that mirrors the kinematic structure and headway of the stick insect.Appropriate calculation for the kinematic model of the six legged strolling robot has been proposed and recreation and examination of various kinds of steps for various rates of the robot body have been performed.The strolling exhibitions of the robots are carefully identified with movement of the tip of every leg system and in this paper this movement is dissected regarding direction and speed of leg tip and furthermore during quickening,transient and consistent state condition for various velocities of the robot body.It has been seen that walk is important to guarantee static steadiness of the robot during walking.Time charts have been drawn which show that the withdrawal speed fluctuates straightly with increasing speed prior to arriving at consistent state condition to balance it to robot speed.

Arne Roennau[22],et.al., (2010) built up a six legged robot LAURON IVc(Legged Autonomous Robot Neural Controlled) motivated from stick bug however with an extra joint which conventional six legged robots don't have,so as to give an extra level of opportunity so the foot would now be able to be arranged in an ideal point to the ground and to extend the workspace of our leg contrasted with past leg ages to make it similar to that of a stick insect. The extra fourth revolute joint makes the kinematic structure more excess due to which the reverse kinematics is tackled in an extremely proficient way.Different potential answers for the extra revolute joint have been obtained and examined.

\section{Other approaches:}

Other than the models and analysis and optimization approaches for the legged frameworks streamlining the referenced in the preceding sub sections, different methodologies have additionally been inconsistently utilized.

Mingfeng Wang [23], et.al., (2014) built up a LARM leg component utilizing an equal mount controller since equal controllers are notable for higher payload capability, stiffness, accuracy and dynamic execution rather than customary chronic manipulators. For portraying the situation behaviour, a recommended movement with an isosceles trapezium direction is used. However the direction of the human-enjoyed stride is an ovoid bend where the straight line section speaks to the supporting stage and the bend fragment speaks to the swinging phase. The bend fragment is then rearranged by straight segments. Each step hover of the movement whose direction is an isosceles trapezium, is isolated into six sections by six esteemed positions where the beginning and the end esteems are given as starting situation of the foot plate. As the three straight actuators are driven under a position and speed open circle control, the endorsed removals have been processed in MATLAB climate and afterward changed into ARDUINO ahead of time programming, the relocations of three direct actuators are needed from which the speeds and increasing velocities are acquired and plotted. Experimental results and mathematical calculations are then kinematically broken down for the motivations behind activity execution assessment and engineering plan portrayal. 
Jenq-Shyong Chen [24], et.al., created a novel Parallel Kinematic Mechanism (PKM) called Cartesian-Guided Tripod (CGT) that has two sorts of practical autonomous legs, the driving utilitarian leg and the coordinated Cartesian directing/metrology useful leg. Due to the detachment of metrology work from the drive instruments, the metrology circle of CGT is inoculated to the mathematical, thermal, and power incited blunders of the drive systems. Another property of this CGT is that the kinematic boundaries can be auto adjusted on the line. CGT has special arrangements for the reverse and forward kinematic investigation. These special arrangements also permit framework determinations, for example, the impression/workspace proportion, greatest Cartesian speed/quickening ratio and even firmness, be effectively identified with the machine part details. The CGT drive instrument can be separated into two fundamental sorts: sliding leg and extending leg. The extending leg CGT has the higher edges in the kinematic classes than the sliding one, but the sliding leg CGT has better dynamic firmness and better static inflexibility.

\section{Conclusion:}

During the most recent years, mechanical frameworks ready to move by implies like those found in the nature have been developed consistently.Legged robots present critical favourable circumstances over the wheeled and tracked ones since they permit motion in landscape out of reach to the customary wheeled and tracked vehicles,since they need not bother with a constant help surfacnspite of the fact that the alluded aspects demonstrate that legged headway is beneficial when analyzed with conventional motion frameworks, it ought to be remembered that in their current state of developments, these robots actually experience the ill effects of gigantic impediments, as they show low speeds, as they are hard to manufacture and are in need of complicated control calculations. Furthermore the present mechanisms are hefty since they need countless actuators for moving different DOF legs, which are also associated with huge energy utilization.

Later on vehicles embracing headway through fake legs turn into a productive shipment mode,that can rival other traditional shipment and transport modes. Nonetheless as expressed beforehand,in their current situation with advancement,there are still a few viewpoints that need to addressed and improved.One of the significant disadvantages of this innovation is its higher energy utilization.

It has been verified that the headway effectiveness of the current legged frameworks is extremely low in contrast to living creatures or wheeled robots.Subsequently a more profound consideration ought to be committed to the enhancement of such frameworks either during its research and development or during its activity in particular to walk determination and characteristic variations to territory and velocity conditions.

The paper presented a survey of strategies that have been used in the analysis and optimization of the bio inspired legged robots and other equivalent methods and approaches.

\section{Acknowledgements:}

We would like to acknowledge Prof Jaspreet Singh,Department of Mechanical Engineering,Lovely Professional University for his constant guidance throughout the course of our work.

\section{Declaration of Interests:}

The authors declare that they have known no competing financial interests or personal relationships that could have appeared to influence the work reported in this paper.

\section{References:}

[1] Liang C, Ceccarelli, M. \& Takeda, Y, “Operation analysis of a Chebyshev-Pantograph leg mechanism for a single DOF biped robot", Front. Mech. Eng. 7,357-370 (2012), doi:10.1007/s11465-012-0340-5.

[2]Mingfeng Wang and Marco Ceccarelli, "Design and Simulation of Walking Operation of a Cassino Biped Locomotor",Article in New Trends in Mechanism and Machine Science,2015, pages 613-621,ISBN: 978-3-31909411-3. 
[3] Hernández-Santos C, Rodriguez-Leal E, Soto R, Gordillo JL, “ Kinematics and Dynamics of a New 16 DOF Humanoid Biped Robot with Active Toe Joint",International Journal of Advanced Robotic Systems, November 2012, doi:10.5772/52452.

[4]Maiorino Andrea and Muscolo Giovanni Gerardo, "Biped Robots With Compliant Joints for Walking and Running Performance Growing" in Frontiers in Mechanical Engineering ,Volume-6 ,2020,Pages:11 ,doi:10.3389/fmech.2020.00011, ISSN:2297-3079.

[5]Rahul R Thavai and Shishirkumar N Kadam, "Kinematic,Dynamic Modeling and Simulation of Biped Robot", International Journal of Science, Engineering and Technology Research(IJSETR), Volume 4, Issue 4, April 2015.

[6 ]Jose A Teneiro Machado, "Kinematic analysis and modelling of biped locomotion systems", in Journal of the Brazilian Society of Mechanical Sciences, September 1999,doi:10.1590/S0100-73861999000300003.

[7] V, Janardhan and R, Prasanth Kumar (2013), "Kinematic analysis of biped robot forward jump for safe locomotion" in 16th National Conference on Machines and Mechanisms, 18-20 December 2013.

[8] Sarkar Nilanjan, Kljuno Elvedin and Williams, Robert L, 2010, "Humanoid Walking Robot: Modeling, Inverse Dynamics, and Gain Scheduling Control”,SN -1687-9600,doi:10.1155/2010/278597,Journal of Robotics, Hindawi Publishing Corporation.

[9] Shriyam, Shaurya \& Mishra, Anand \& Nayak, Debasish \& Thakur, Atul. (2014), “Design, Fabrication and Gait Planning of Alligator-Inspired Robot", International Journal of Current Engineering and Technology,567-575. 10.14741/ljcet/Spl.2.2014.

[10] Takuma Nemoto,Rajesh Elara Mohan,and Masami Iwase (2015), "Rolling Locomotion Control of a Biologically Inspired Quadruped Robot Based on Energy Compensation",Hindawi Publishing Corporation,Journal of Robotics,Volume 2015,Article ID 649819,10 pages,https://dx.doi.org/10.1155/2015/649819.

[11]Sheba, Jaichandar \& Martinez-Garcia, Edgar \& Mohan, Rajesh Elara \& Le, Tan-Phuc. (2015), “Design and evaluation of reconfigurable Klann mechanism based four legged walking robot", 1-5. 10.1109/ICICS.2015.7459939.

[12]Spadaro Francesco Tracuzzi and Muscolo Giovanni Gerardo , "Influence of Compliant Joints in Four-Legged Robots" in Frontiers in Mechanical Engineering, 2020,Volume-6,pages:16,doi: 10.3389/fmech.2020.00016 ,ISSN:2297-3079.

[13]Li Y, Fish F, Chen Y, Ren T, Zhou J, "Bio-inspired robotic dog paddling: kinematic and hydro-dynamic analysis" Bioinspir Biomim,2019,Sep 13;14(6):066008. doi: 10.1088/1748-3190/ab3d05. PMID: 31430730.

[14]Jizhuang F, Qilong D, Qingguo Y, Yi W, Jiaming Q, Yanhe Z, "Biologically inspired swimming robotic frog based on pneumatic soft actuators",Bioinspir Biomim,2020,May 27;15(4):046006. doi: 10.1088/1748-3190/ab835a. PMID: 32209752.

[15]Sousa Daniel \& Botelho, Wagner \& Marietto, Maria \& Ferreira, João \& Pimentel, Edson,(2017), “TB-Horse II: Design And Analysis of a Bio Inspired horse based on the breed Mangalarga Marchador", 65-72. 10.1142/9789813231047_0011.

[16]Conghui Liang, Marco Ceccarelli, and Giuseppe Carbone,2009, “ A novel biologically inspired tripod walking robot"in Proceedings of the 13th WSEAS international conference on Systems, World Scientific and Engineering Academy and Society (WSEAS), Stevens Point, Wisconsin, USA, 83-91.

[17]Shahriari, Mohammadali \& Ghaemi Osgouie, Kambiz \& Osguie,. (2014), "Kinematic and Gait Analysis Implementation of an Experimental Radially Symmetric Six-Legged Walking Robot",10.13140/2.1.2192.0003.

[18] Shibhendu Shekhar Roy and Dilip Kumar Pratihar, "Analysis of Six legged Walking Robots",14th National Conference on Machines and Mechanisms (NaCoMMO9),December 17-18,2009. 
[19] Servet Soyguder and Hasan Alli (2007), "Design and prototype of a six legged walking insect robot",in Industrial Robot 34(5):412-422,doi:10.1108/0143991071074412.

[20] Asif, Umar\& Iqbal Javaid \& Khan ,M. Ajmal. (2011), " Kinematic analysis of periodic continuous gaits for a bio-mimetic walking robot" 9th IEEE International Symposium on Safety, Security, and Rescue Robotics, SSRR 2011.10.1109/SSRR.2011.6106784.

[21]G.Figliolini,S-D.Stan and P.Rea, "Motion Analysis of the Leg Tip of a Six-Legged Walking Robot",12thIFToMM World Congress,Besancon (France),June18-21,2007.

[22]Arne Roennau and Rudiger Dilmann (2010), "Design and Kinematics of a biologically inspired leg for a six legged walking machine",3rd IEEE RAS \& EMBS International Conference on Biomedical Robotics and Biomechatronics,Tokyo,2010,pp.626-631.doi: 10.1109/BIOROB.2010.5626328.

[23]Wang, Mingfeng \& Ceccarelli, Marco \& Carbone, Giuseppe. (2014), “ Experimental experiences with a LARM tripod leg mechanism", 10.1109/MESA.2014.6935547.

[24]Jenq-Shyong Chen, Wei-Yao Hsu, "Design and analysis of a tripod machine tool with an integrated Cartesian guiding and metrology mechanism",Precision Engineering, Volume 28, Issue 1,2004,Pages 46-57, ISSN 0141-6359,https://doi.org/10.1016/S0141-6359(03)00073-4. 